\title{
FLYING SATS HIGHER VOLUME OPERATIONS: TRAINING, LESSONS LEARNED, AND PILOTS' EXPERIENCES
}

\author{
Sheila Conway, Dan Williams, Catherine Adams, Maria Consiglio, and Jennifer Murdoch \\ NASA Langley Research Center, Hampton, VA USA 23681
}

\begin{abstract}
Developments in aviation, including new surveillance technologies and quicker, more economical small aircraft, have been identified as driving factors in a potential expansion of the use of non-towered, non-radar airports. The Small Aircraft Transportation System (SATS) project has developed the Higher Volume Operations (HVO) concept that enables pilots to safely arrive and depart these airports in instrument conditions at an increased rate as compared to today's procedures. This is achieved by transferring some traffic management tasks to centralized, ground-based automation, while assigning others to participating pilots aided by on-board tools. This paper describes strategies and lessons learned while training pilots to fly these innovative operations. Pilot approaches to using the experimental displays and dynamic altering systems during training are discussed. Potential operational benefits as well as pit-falls and frustrations expressed by subjects while learning to fly these new procedures are presented. Generally, pilots were comfortable with the procedures and the training process, and expressed interest in its near-term implementation.
\end{abstract}

Nomenclature

$\begin{array}{ll}A M M & =\text { Airport Management Module } \\ A T C & =\text { Air Traffic Control } \\ A T P & =\text { Airline Transport Pilot } \\ C T A F & =\text { Common Traffic Advisory Frequency } \\ D F & =\text { Departure Fix } \\ F A F & =\text { Final Approach Fix } \\ F O I & =\text { Fundamentals of instruction } \\ F T D & =\text { Flight Training Device } \\ F T E & =\text { Flight Technical Error } \\ G A & =\text { General Aviation } \\ H S I & =\text { Horizontal Situation Indicator }\end{array}$

$\begin{array}{ll}\text { HITL } & =\text { Human in the Loop } \\ H V O & =\text { Higher Volume Operations } \\ I A F & =\text { Initial Approach Fix } \\ I F R & =\text { Instrument Flight Rules } \\ I M C & =\text { Instrument Meteorological Conditions } \\ M A H F & =\text { Missed Approach Holding Fix } \\ M F D & =\text { Multi-Function Display } \\ \text { NAS } & =\text { National Airspace System } \\ \text { SATS } & =\text { Small Aircraft Transportation System } \\ S C A & =\text { Self-Controlled Area } \\ V F R & =\text { Visual Flight Rules }\end{array}$

\section{Introduction}

$\mathrm{T}_{\mathrm{i}}$ HE ability to conduct concurrent, multiple aircraft operations in poor weather at virtually any airport offers an important opportunity for a significant increase in the rate of flight operations, a major improvement in passenger convenience, and the potential to foster growth of operations at small airports. The Small Aircraft Transportation System (SATS) Higher Volume Operations (HVO) concept is designed to increase capacity at the 3400 non-radar, non-towered airports in the United States where operations are currently restricted to "one-in/oneout" procedural separation during low visibility or ceilings.

When instrument meteorological conditions (IMC) restricts operations to Instrument Flight Rules (IFR) at nontowered, non-radar airports, air traffic control (ATC) uses procedural separation that restricts operations to only one approaching or departing aircraft at a time - the "one-in/one-out" paradigm. While procedural separation is safe, it severely limits the operational throughput at these airports. The HVO design uses distributed decision-making integrated within current and planned near-term National Airspace System (NAS) infrastructure. The underlying design emphasizes simplicity from both a procedural and systems requirements standpoint: Clearly, any additional ATC or pilot workload must be minimized, and enroute procedures must be compatible with today's ATC system.

Pilots flying HVO require supplemental procedure and knowledge training to ensure they are qualified to communicate appropriately, understand the HVO procedures, and interface with on-board and off-board automation. Researchers believe that procedure and knowledge training specific to SATS HVO can be a logical extension of the instrument rating without requiring the addition of training pilots to use new skills. 
Several experiments with instrument-rated pilots were conducted to assess if SATS HVO could be flown safely and proficiently with acceptable workload. Based on positive results, it seems SATS HVO can be flown with similar, if not lower workload, flight technical error (FTE), and better situation and navigation awareness than the procedures in use today. Additionally, SATS HVO training requirements appears to integrate well with federallymandated instrument flight training and recurrency programs and commensurate NAS operations.

\section{Background}

\section{A. SATS HVO Operational Concept Overview}

Key to the SATS HVO concept is a distributed decision-making environment that assumes major decisionmaking responsibility for the pilot, and resource mitigation for ground-based automation known as the airport management module (AMM). The concept utilizes a newly defined flight operations area called a Self-Controlled Area (SCA), established during periods of IMC around "SATS designated airports" (i.e., non-towered, non-radar airports). Within the SCA, pilots, using new procedures and airborne information systems would have the ability and responsibility to maintain separation between themselves and other similarly equipped airplanes, while sharing information with the AMM that in turn sequences approach traffic.

\section{Normal Operations}

Aircraft will approach a SATS airport on an IFR clearance granted by ATC to a transition fix above the SCA. This fix is also an initial approach fix (IAF) for an instrument approach procedure*. Prior to reaching the fix, the pilot requests a landing assignment from the AMM through their onboard system. The AMM responds with the SCA entry procedure (standby, vertical or lateral), relative sequence information (follow <callsign>), and missed approach hold fix assignment (MAHF, e.g. ANNIE or CATHY). The AMM only sequences arrivals (including missed approach aircraft), not departures.

All aircraft on approach (including a missed approach segment) are guaranteed a holding slot at one of the MAHFs, hence the maximum number of arrivals for an SCA is limited by the number of holding slots. Nominally, up to four arriving aircraft are allowed in the SCA before denying entry (issuing a "STANDBY"), though this constraint can be affected by local airspace restrictions ${ }^{\dagger}$. By following entry assignments, i.e. the "descend to lowest available altitude" HVO procedure, ensuring appropriate spacing from any lead aircraft, and flying their approach to instrument flight standards, pilots are deconflicted from other arriving traffic.

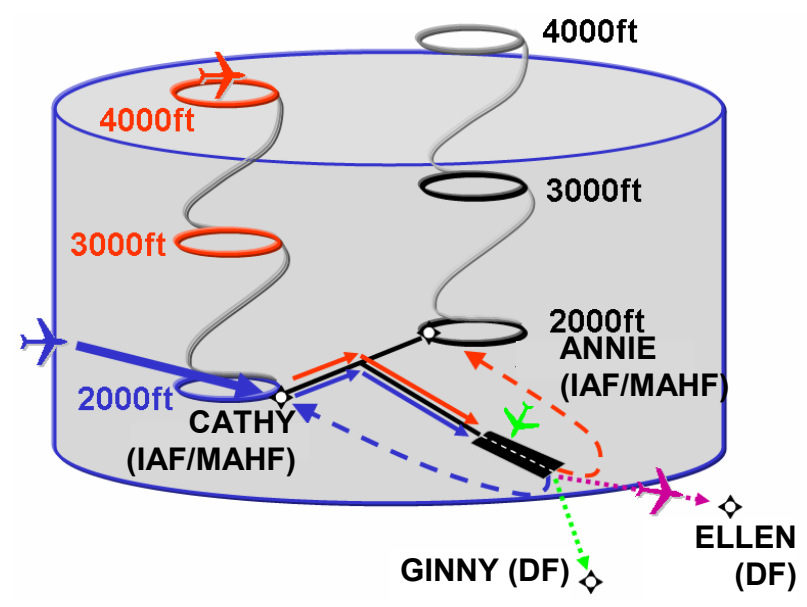

Figure 1. SATS HVO Example

\footnotetext{
* GPS-T instrument approach procedures were chosen as the basis for this concept, although other instrument approach procedures could be used.

${ }^{\dagger}$ Fig 1 shows the nominal approach design with 4 potential holding segments, 2 altitudes at each IAF. Differing numbers of IAFs (e.g. some approaches may only have one) or altitude constraints (e.g. 3000' available below the enroute structure, affording 3 holding altitudes at the IAFs) can increase or inhibit holding segments.
} 
Figure 1 illustrates many of the features of the SATS HVO concept, including arrivals (in Red and Blue) to the IAFs with alternating missed approaches, and departures (in Green and Purple) to the Departure Fixes (DFs):

Blue - entering the SCA having coordinated descent with ATC when no other aircraft assigned to CATHY, missed approach is blue dashed path, AMM returned:

LATERAL entry, follow NONE, missed approach CATHY

Red - having arrived by ATC instruction to transition fix above SCA at 4000ft with one other CATHY assignment, the AMM returned: Vertical entry, follow BLUE, missed approach ANNIE

Purple - departing SCA via departure procedure and contact ATC prior to DF

Green - released by ATC to depart (within departure window); holding short and using on-board tools to find open slot in arrival stream to take the runway and depart

Pilots that are given a "standby" sequence can track the number of aircraft in the SCA to estimate their delay as they continue to their clearance limit, the transition fix at an altitude above the SCA, and hold. When the pilot receives an AMM entry message with sequence and missed approach information, the pilot checks for an available holding altitude, and will request descent from ATC. The pilot can then determine if further descent is prudent by following the "lowest available altitude" procedure at the IAF, (clearing for traffic below is the pilot's selfseparation responsibility in the SCA). Pilots initiate their approach once adequate spacing behind the lead aircraft has been met (determined through either a generic rule-based spacing procedure, i.e., safe for all combinations of aircraft performance, or by using an on-board self-spacing tool). The AMM reserves a holding slot for assigned missed approaches. A pilot executing a missed approach would climb to the "lowest available altitude" at their assigned MAHF and would be sent a new arrival sequence.

For SATS departures, pilots will file flight plans including a SATS departure procedure to a departure fix (DF, i.e., Figure 1 ELLEN or GINNY). Just as in today's non-radar environment, they should expect a clearance void time and potentially a release time restriction as part of their IFR clearance. This affords seamless integration with today's instrument flight operations. Within this ATC departure window, they will use on-board information/tools to deconflict themselves with landing traffic, e.g., ensure no arriving aircraft within $5 \mathrm{~nm}$ of the airport. The pilot would then depart and contact ATC according to the departure procedure before entering ATC controlled airspace.

\section{Off-Nominal Operations}

Baxley et al ${ }^{1}$ describe three categories of off-nominal situations that may occur in a future HVO environment: routine, such as a change of landing approach direction or pilot operational errors; equipment malfunctions, such as a loss of an aircraft's communication system; and emergency situations, such as a priority request for an emergency landing. They developed procedures to handle a number of these situations.

Two of these procedures were tested in a piloted simulation study ${ }^{2}$ : procedures to handle cancellation of approach requests with transitions to visual flight rules (VFR), and priority requests from approaching aircraft. Priority landing requests allow pilots to land ahead of others in the sequence. Cancellations of approach requests allow participating pilots to weather permitting, transition to a visual approach if desired. While there is substantial work to be done in this area, the choice of off-nominal procedures for this first study was based on the limited scope of the study, the expected likelihood of VFR transitions, the relative importance of priority landings, and their foreseen potential to influence the tenets of HVO.

\section{B. Validating the SATS HVO Concept and the Use of Piloted Studies}

The SATS HVO research team developed and applied a four phase process to design and validate the HVO concept of operations (CONOPS), depicted in Figure 2.

Phase one involved HVO CONOPS development. The key safety properties of the HVO CONOPS were established by mathematical verification methods based on formal logic and theorem proving ${ }^{3}$. This study began formally verifying that self-separation can be maintained when pilots adhere to the HVO procedures and AMM logic is appropriately implemented.

Phase two involved the development of computer simulations. The AMM function and associated algorithms were verified and validated using a representative set of HVO scenarios.

Phase three, the primary subject of this paper, determined if HVO functionality and procedures were acceptable to pilots. Human-in-the-loop (HITL) scenarios were developed that compared the SATS HVO CONOPS to the onein/one-out procedural control environment available today (Baseline) and addressed the two questions:

- Can pilots safely and proficiently fly the airplane while performing SATS HVO procedures?

- Do pilots perceive that workload, while using HVO procedures and tools, is no greater than flying in today's system? 


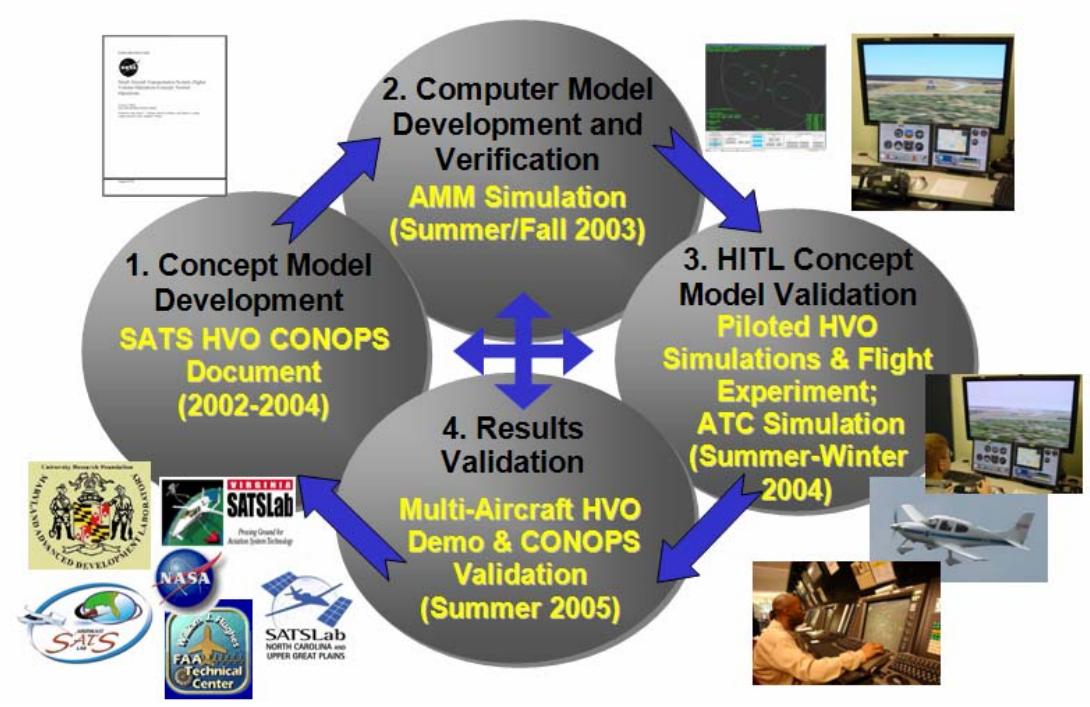

Figure 2. HVO Validation

Groups of instrument rated subject pilots were evaluated and provided feedback as they flew the scenarios in experiments using progressively higher fidelity simulation, from a medium fidelity general aviation (GA) computer simulation to the Cirrus SR22 aircraft in flight. Consistent early results across the various simulation platforms provided confidence in the validity of the simulation results, so later studies of the HVO concept were conducted with the simulation platform. Subsequently, two additional simulation experiments determined if non-normal procedures were acceptable to the pilot as well as the procedure support automation developed by NASA researchers ${ }^{4}$, and the necessity of advisory messaging on the Multi-Function Display (MFD) ${ }^{5}$. Also, an ATC simulation study was completed to assess controller acceptability of the concept model ${ }^{6}$.

Phase four was a proof-of-concept public demonstration of six SATS Lab aircraft flying the SATS HVO procedures in the 2005 SATS Technology Demonstration held in Danville, Virginia. All four phases provided feedback to the improvement of the SATS HVO CONOPS and ultimately toward recommending a viable way to improve upon the one-in/one-out procedure in place in the NAS today.

\section{The Need for Subject Pilot Training}

HVO procedures are based upon current IFR procedures, but they are still new. While the aircraft control task for flying the approach itself is much akin to traditional instrument flight, HVO require monitoring dynamic messaging elements on a multi-function display as well as a substantial awareness of traffic. Because of the novel application of datalink messaging and responsibility for traffic avoidance, pilots have new tasks and must receive specialized training to understand and assume their responsibilities in entering/exiting and operating within the SCA. These responsibilities include: separating from other SCA aircraft, following the AMM derived aircraft landing sequence, self-initiating the approach (or departure); and exchanging HVO information with ATC, the AMM, and other participating pilots. For both the simulation and flight experiments, researchers spent several hours training subjects in these unique tasks.

Deliberately, subjects with a common level of inexperience were selected, i.e., none had familiarity in flying the SATS GA simulator and the Cirrus SR22. The first step in training was to familiarize the pilots with the flight controls and aircraft performance. After aircraft controls training, pilots were oriented to the HVO functionality and procedures.

\section{Subject Pilot Selection}

In choosing a subject pool for the validation experiments, researchers identified criteria for pilots who were capable, but not overly experienced. Although not tested, it was postulated that if the low-time instrument rated subject pool validated the concept positively, then more experienced pilots would do the same. Subject pilot criteria included low total flight time (less than 1000 flight hours), a high performance endorsement, an instrument rating, and legal currency to fly IFR. Additionally, they were required to have experience with a Horizontal Situation Indicator (HSI) and with flying GPS-based instrument approaches. None of the participants had previously flown a Cirrus SR22 aircraft, flown for the military, or worked as a flight crewmember for an air carrier within the last year. 


\section{A. Basic Instrument Flight Qualifications}

Participating subject pilots were required to have an instrument rating, ensuring instrument flight knowledge, and to be instrument current, ensuring recent flight experience and proficiency. This section provides an overview of federal requirements for the instrument rating which allows a pilot to fly under IFR.

\section{Basic Licensure}

The IFR course is a rigorous training program where a pilot learns to fly by reference only to instruments to control and guide the aircraft - maintaining level flight, executing turns, climbs and descents and combinations of those maneuvers. The pilot learns to take off, track the aircraft's position solely with reference to the instrumentation, and land the aircraft after acquiring the airport visually at a point designated typically before or at the runway threshold.

The Federal Aviation Administration (FAA) is charged with the responsibility of developing and maintaining regulations pertaining to the licensure of pilots of varying level of competency for different aeronautical disciplines. Pilots cannot fly in IMC in controlled airspace unless under IFR and licensed to do so by an FAA designated examiner as defined by 14CFR61.65 . IFR candidates must hold at least a private pilot's license in the category of aircraft to be flown (i.e. aircraft or helicopter). In order to be eligible for a flight exam, a pilot must pass a knowledge test and receive appropriate training in instrument flight during both enroute and approach phases that includes a combination of 40 hours of simulated or actual instrument conditions.

\section{Recurrent training}

Regulation 14CFR61.57 $57^{8}$ specifies the requirements to maintain instrument currency. Pilots must fly at least six instrument approaches, execute holding procedures, and intercept and track courses in the last 6 months in either an aircraft or an approved flight training device (FTD) representative of the category and class of aircraft for which they seek these privileges. If the pilot has not met these requirements, an instrument proficiency check in the same category and class aircraft by an examiner or instructor is required.

\section{B. Flight Simulation Experience}

All of the subject pilots had some simulator experience. Many of them were students in flight training programs where FTDs are frequently used. These pilots were very familiar with flight simulators, having used them extensively as devices to expand their knowledge and experience.

\section{Training Principles}

There were three primary training principles that influenced the flight training strategies for these SATS HVO experiments: 1) All subjects were experienced, current instrument pilots, affording the opportunity to teach "differences" rather than comprehensive SATS HVO instrument flying. 2) The fundamentals of instruction would be useful in introducing a new concept founded in traditional instrument flight. 3) Development principles and the demonstration-performance teaching method were employed to introduce and ensure adeptness in three major portions of the subjects' tasks, namely aircraft control, standard instrument flight, and HVO operations. Differences training, the fundamentals of instruction and development principles are all general instructional concepts that have been tailored specifically for flight training by the FAA.

\section{A. Differences Training}

One of the primary goals of the SATS HVO concept was to leverage (as much as practical) existing instrument flight procedures. By requiring all subject pilots to be familiar with these baseline instrument procedures, and the procedures being only marginally distinct, course content became a matter of explaining and practicing the new HVO material and its relation to standard, instrument flight. This concept of differences training was modeled after professional training requirements for augmenting basic skills of commercial air carrier flight crews. Federal Aviation Regulations Parts 121.401Traning Program: General and 121.418 Differences Training ${ }^{9}$ require ground instruction applicable to assigned duties and responsibilities, and flight training in each appropriate maneuver or procedure. They assume that non duty-specific flying skills (appropriate for a pilot's ratings) are already established. These same requirements seem prudent for non-Part 121 operations as well, and were the basis of the SATS HVO differences training.

However, unlike what might be expected of pilots flying in a part 121 operation, the subject pilots in the HVO experiments had substantially varying experience. While pilots in a part 121 operation have to continually pass a gauntlet of check rides and generally fly similar IFR flights regularly, the subject pools for HVO were only required to be instrument current, ensuring only a minimal amount of flying within the past six months and recent 
experience with limited approach types. In fact, most pilots fly in actual IMC only occasionally, making recent experience beyond legal instrument currency minimal for even seasoned Airline Transport Pilots (ATP's) ${ }^{10}$. IFR experience, quantity, quality, diversity and recency, all influence a pilot's overall instrument skill level. Anecdotally, total instrument time and ratings seemed to be less related to subjects' comprehensive instrument flight skill and comfort levels than were other factors.

The largest single factor in practicing HVO differences training was the typical airport facility the subjects frequented. For those who flew at a towered airport, particularly a busy approach-controlled facility, these selfresponsive operations were largely foreign. These subjects, often with intense, concentrated part 141 flight school experience, were accustomed to controller intervention in nearly all stages of flight, and typically had not experienced a large diversity of operations. This was also seen with certain transport category pilots who did little GA flying. As an example, some subjects that generally flew only from towered airports were not in the habit of checking for traffic on the final approach before taking the runway for departure. Some had never departed IFR from an uncontrolled field using a void time clearance since initial training. On the other hand, other subjects had substantial experience in operating from non-towered airports outside a radar service area, and were accustomed to clearance void times, appropriate self-announcing on a traffice advisory frequency, etc. For this reason, while differences training was the starting strategy, in the end, many relevant, standard IFR and VFR procedures that were leveraged for HVO were also reviewed to "level the playing field" across subjects.

\section{B. Instructors: Remember your FOI?}

The FAA's fundamentals of instruction (FOI) ${ }^{11}$ were used as the guidelines for the training program. As any FAA Certified Flight Instructor can attest, the FOI describes how students learn, the stages or levels of learning, and how to help students achieve the more advanced stages of application and coordination knowledge rather than only rote understanding. Any rated instrument pilot has demonstrated instrument flight knowledge across many subjects, but the level to which they truly understand the subjects cannot always be determined. Additionally, as time passes, their perceptions of instrument flight change, sometimes skewed by a limited exercise of only certain instrument procedures. By reviewing the foundational procedures, quizzing the subjects, and practicing them before the differences training, the subjects were all brought to the correlation level (shown in Fig. 3) necessary as context for the HVO training.

\section{Development Principles and the Demonstration-Performance Method}

Once it came time to design the actual training materials and plan the training sessions, the development principle and the demonstration-performance method of teaching were employed. Since all of the subjects were unfamiliar with the flying characteristics of the PC-based simulation and/or the Cirrus test aircraft, they were first given the opportunity to practice flight maneuvers and taught appropriate technique, e.g. power and flap settings for particular descent profiles, using demonstration-performance techniques. They were then given the opportunity to practice standard instrument approaches. Once comfortable with these everyday flying maneuvers, they were given ground instruction on the concept, and then again by demonstration-performance, an opportunity to practice the HVO flight procedures with supervision. In this progression supported by development principles ${ }^{12}$, basic instrument flying skills were mastered before introducing the new HVO material, affording students a learning environment with minimal distraction.

\section{SATS Specific Concerns}

The single biggest difference between this training and other instrument flight instruction was ensuring that pilots understood that HVO represents a fundamental change in roles and responsibility in instrument flight. Unlike other instrument flight operations, SATS HVO requires pilots to be aware of and follow separation minima. Under most traditional instrument flight operations, these concerns are the purview of controllers. However, there is precedence for such responsibility in VFR flight and the IFR visual approach procedure, where pilots have responsibility to maintain "safe" separation from traffic. 
The primary difference in HVO is that there is a formalized definition of "safe" (by which most visual measures would be very conservative). For these initial experiments, there was no attempt to optimize these safety-related parameters. Rather, standard radar separation rules were used. These in turn were not necessarily shared with the pilots in the same manner they might have been with a controller. Rather, pilots were given rules-of-thumb to follow that would in turn conservatively ensure adherence to these legal minimum separations.

For example, rather than considering along track distance, actual distance and closure, pilots following traffic on an approach were instructed to not leave holding and begin their own approach until their follow traffic was inside the final approach fix (FAF). Because of the speed class limitations for approaches and the resultant limited potential for speed differences among approaching traffic, this spacing buffer could accommodate closure between aircraft and provide for minimum or greater spacing at the closest point of approach. While not the most efficient use of the airspace in all situations, this rule-of-thumb simplified the somewhat complex geometry and rule set that would otherwise have been necessary for the pilot to consider. As an aside, there are other alternatives to this solution that have been explored in SATS and elsewhere. They involve more data sharing between aircraft, and the use of relative control between aircraft for more efficient spacing on approach.

\section{E. Commonality between Simulation and Flight Experiments}

Figure 4 shows the GA simulator and the Cirrus SR22's instrument panel used for the experiments. They used common software across platforms to drive the research MFD, including a moving map, and the Horizontal Situation Indicator (HSI) displays. Variation between the simulation and flight profiles was deliberately minimized so as not to alter the experiment objectives or hypotheses. Pilots in both simulation and flight were tasked to meet FAA instrument rating practical test standards criteria.

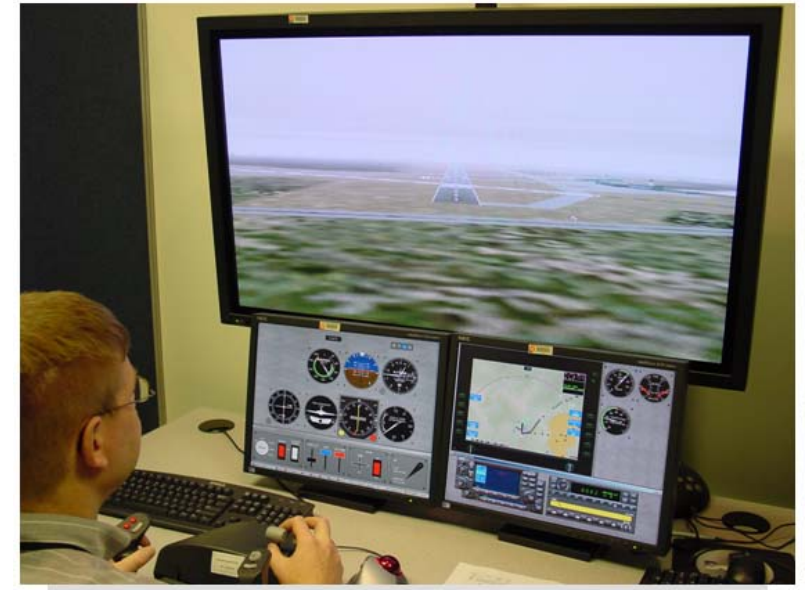

Simulation Experiment Platform GA Simulator, similar to Cirrus SR22
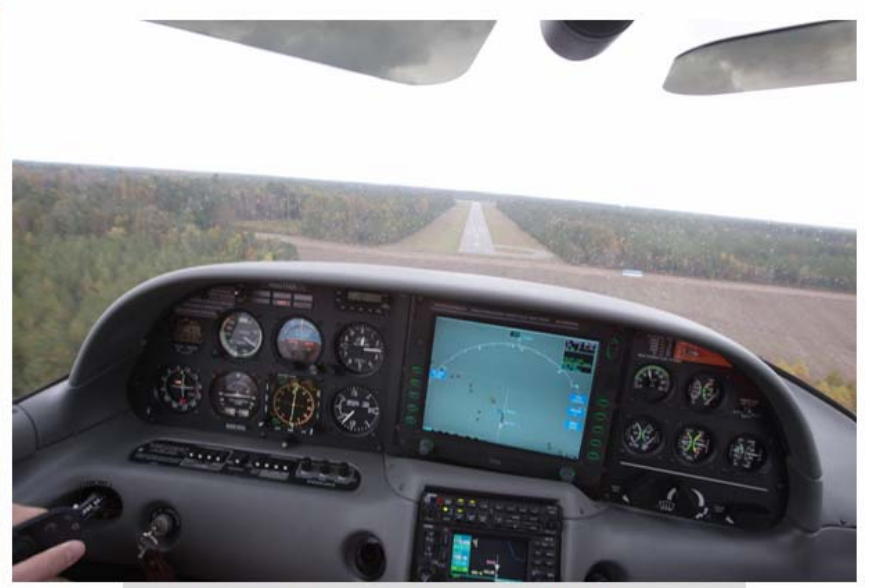

Flight Experiment Platform Cirrus SR22

Figure 4. HVO Experiment Platforms (note commonality of HSI and MFD)

The Project's HVO validation process meant that the HVO flight experiment was flown to validate pilot acceptability of a subset of the HVO simulation scenarios. A common pool of pilots was used. Fifteen pilots flew the HVO Simulation Experiment, and twelve of those pilots flew the HVO Flight Experiment. This reduced training requirements for the HVO Flight Experiment and allowed pilots to progress logically from hand-flying a medium fidelity GA simulator to the Cirrus SR22 aircraft.

\section{F. Experimental Considerations}

Experimental Disclosure

One of the considerations in preparing training materials was how much experimental detail to disclose to the subjects. Best scientific practices (and federal law) requires that all human research subjects to be informed of "the purposes of the research .... a description of the procedures to be followed, and identification of any procedures which are experimental" ${ }^{13}$ For the HVO experiments, all federal guidelines for subject protection were exercised 
(ensured by an Institutional Review Board) with the exception of full disclosure: While subjects were informed of the intent of the research, they were not aware of the experimental hypotheses because this information may have skewed their subjective responses to the experiential questionnaires. However, the experiment included no undisclosed risk to the subjects as well as a debriefing and dissemination of research results, fulfilling subject protection obligation while maintaining scientific utility.

\section{How Much Training is Enough?}

Another consideration was the necessary level of detail to present to the subject pilots. Analogous to today's IFR environment where pilots may not know all the reasons behind a particular ATC clearance but can still respond to a specific request for action, pilots did not need to know all the details necessary to design or even execute comprehensive HVO procedures, but rather only a select subset of them. More knowledge is not always helpful, particularly in nominal situations, because the pilots and controlling authorities have different data and different objectives. However, in other situations, particularly off-nominal cases, knowing more information regarding the reasoning behind a procedure could afford a pilot or controller an opportunity to synthesize options that still meet the intent of the procedure but are outside standard operating procedure.

For the SATS HVO experiments, pilots were given operational detail similar to that included in an instrument ground school curricula as well as operational rules-of-thumb to provide sufficient, safe traffic separation. They were not trained on the inner-workings of the automated equipment, or the detailed actual values of minimum separation control criteria, as their non-optimizing operations did not, by design, require this level of system knowledge. In fact, the necessity for pilots to recall and apply controller-level knowledge of separation strategies and minima was assumed to exceed workload capacity when combined with real-time flying tasks.

As it would be in an actual instrument flight training program, additional details were introduced to the pilots in the off-nominal experiment training sessions after building basic skills and knowledge.

\section{Can Insufficient Training be Differentiated from Piloting Skill?}

While it is true that all of the subject pilots were legally qualified and current instrument pilots, there was a marked range of skill and confidence amongst them. In the first experiments, pilots were all given identical training. In subsequent experiments, researchers wanted to explore the notion of one-on-one pilot queries: a "phase check" before the data runs. During the pilot queries, at then end of each major training block, researchers questioned pilots as they flew a scenario on procedures, the meaning of the messages, and their strategies for spacing and flying the approaches. Using this technique, the researchers could gauge if each subject was sufficiently familiar with HVO and their basic piloting skills. In all but two cases, pilots were able to perform the scenarios without missing cues or forgetting the procedures, implying sufficient training. In these cases, a small amount of additional instruction and practice added to the group's training session brought all the subjects to the correlation learning-level, necessary to handle off-nominal situations.

\section{Mitigation Strategies - Experimental Design}

While every effort was made to ensure sufficient training, it was likely that pilots would continue to improve their performance with practice, or experience a "learning effect." An emphasis in the experimental design was then to minimize the influence of learning effects so that subject inexperience would not impact measuring relative FTE: Comparing performance of the HVO concept against that of baseline (conventional) IFR procedures required the establishment of a matrix for a randomized partial factorial presentation of baseline and HVO scenarios. The various scenarios were designed to give pilots exposure to departures, single aircraft approaches, multiple aircraft approaches and missed approaches under both conditions of baseline and SATS HVO procedures. The scenarios were presented to the subjects such that on average, some flew certain combinations early in the experiment while other subjects flew them later. Statistically speaking, the randomized presentation of scenarios among the subjects minimized any residual learning effect on the aggregate experimental results.

\section{Training Curricula}

The training was directed towards executing SATS HVO instrument approach and departure procedures to/from a conventionally-controlled airspace environment. The objective was to have a high content validity while, in short order, facilitating the creation of an appropriate mental model for the complex process of flying and simultaneously managing the SATS HVO procedures and commensurate responsibilities.

A building block approach was employed to prepare subject pilots for accomplishing the experiment tasks: The general HVO concept was first presented in a classroom training session, followed by a description of the flying characteristics of the simulated aircraft, and the flight controls and instruments of the simulator. The subjects were then given a hands-on simulator training session to familiarize themselves with the simulated aircraft. Once basic 
flight maneuvers were mastered, they were tasked to manually fly (i.e., without autopilot) the scenarios in IMC using traditional round-dial instruments and a basic MFD with moving map for flight guidance.

The research MFD interface required focused classroom and hands-on training to orient the subjects to its functionality and operation grounded in the context of HVO operations. Subjects were given an in-depth HVO research display classroom training session, and then another opportunity to fly the simulator, exercise the new onboard equipment, and fly the full HVO procedures. During this training and most SATS experimental scenarios, advisory information provided through the MFD included a moving map with navigation information, traffic depiction, AMM sequence information, conflict detection, procedure conformance monitoring, and alerting.

The simulation training prior to the flight training also helped to transfer the knowledge and skills necessary to perform HVO approaches into the flight environment. The emphasis in training the flight experiment pilots who had all previously flown in a simulation experiment was to refresh their knowledge and to introduce them to the specifics of the Cirrus SR22 aircraft, as subject selection criteria prohibited pilots with Cirrus SR22 flight experience. Each evaluation pilot was provided a pre-arrival briefing packet describing the flight experiment, instrument approaches, and maneuver profile. They were reintroduced to the HVO experiment through a researcher review of the training briefing and the experimental displays in a desktop simulator session. A certified Cirrus instructor, serving as safety pilot, provided additional SR22 orientation before flight as well as in-flight: Under safety pilot instruction, each subject pilot assumed control of the airplane en-route to the test area, flew basic instrument maneuvers and executed two practice instrument approaches before data collection scenarios were flown.

\section{Pilot's Reactions}

Researchers observed that the subject pilots were well trained in the HVO procedures and equipment, and were prepared to fly the experimental scenarios once data collection had begun. Some additional minor skill improvement attributable to learning effect was observed, but not quantified because the experimental test matrix was designed to mitigate its influence.

While not recorded or quantified, some pilots were observed to rely on automated decision support more extensively than others, particularly when flying HVO scenarios. This could be an indication of inexperience or curiosity with HVO and on-board systems. However, it could also be an indication that some pilots need more training to ensure understanding of their responsibility within the SCA for self-separation and self-spacing, and appropriate use of their automation in an advisory capacity.

Most pilots very quickly adopted both the specific HVO procedures as well as an appropriate attitude towards assuming their role in this distributed system. However, a few subjects acknowledged that the proposed operations as presented in the scenarios did not explicitly provide for oversight or rule enforcement, akin to VFR flight today, and that they could foresee some abuse or at least "gaming" of the automated systems. A few exhibited such behavior during the less structured off-nominal scenarios, e.g. flying non-standard approaches under VFR to position themselves favorably in an arrival stream. On the whole, most subjects thought that self-policing in combination with appropriate training would be sufficient to govern operations.

As expressed in HVO experiment debriefing comments, the subject pilots agreed that the training they received was sufficient, logical, and a natural extension of their instrument rating. Usability questionnaires indicated that subjects felt that in addition to nominal instrument credentials, HVO-specific training and an instructor endorsement would be sufficient pilot qualifications for HVO. They suggested the training include a combination of ground instruction and flight training, some of which could be conducted in a FTD. In order to achieve a comfortable level of proficiency, they recommended an average of 6 hours of training: 3 hours each of procedures training (FTD or flight) and 3 hours of flying practice approaches. Future research is necessary to establish the minimum legal HVO training required for initial and recurrent training.

\section{Conclusions}

Over the course of developing and validating the SATS HVO procedures, a number of piloted studies were conducted. Training pilots as subjects for these experiments proved to be a straightforward extension of traditional instrument flight instruction. In general, instrument-competent pilots had little trouble acquiring the necessary knowledge or understanding and executing HVO procedures. The most challenging aspect of teaching pilots to fly SATS HVO seems to be developing an appropriate attitude within the pilot towards their new role in traffic separation assurance and more generally towards distributed responsibility for traffic management 


\section{References}

${ }^{1}$ Baxley, Williams, Consiglio, Conway, Adams, "The Small Aircraft Transportation System (SATS) Higher Volume Operations (HVO) Off-Nominal Operations", accepted AIAA ATIO 2005, Washington DC.

2 Consiglio, Conway, Adams, Syed, "SATS HVO Procedures for Priority Landings and Mixed VFR/IFR Operations", accepted 24 $4^{\text {th }}$ Digital Avionics Systems Conference (DASC), Washington, DC. 2005.

${ }^{3}$ Carreno, Gottliebsen, Butler and Kalvala, "Formal Modeling and Analysis of a Preliminary Small Aircraft Transportation System (SATS) Concept", NASA/TM-2004-212999, March 2004

${ }^{4}$ Consiglio et al, 2005

${ }^{5}$ Adams, Consiglio, Conway and Syed, "The Pilot Advisor: Assessing the need for a procedural advisory tool", accepted $24^{\text {th }}$ Digital Avionics Systems Conference (DASC), Washington, DC. 2005.

6 Lohr, Wiliams, Baxley, Abbott and Greco, "Considerations for the Integration of Small Aircraft Transportation System Higher Volume Operations (SATS HVO) in the National Airspace System (NAS)", accepted AIAA ATIO 2005, Washington DC.

${ }^{7}$ Federal Aviation Administration, Federal Aviation Regulations PART 61--CERTIFICATION: PILOTS, FLIGHT INSTRUCTORS, AND GROUND INSTRUCTORS, www.faa.gov, 14CFR61.65

${ }^{8}$ ibid, 14CFR61.57

${ }^{9}$ Federal Aviation Administration, Federal Aviation Regulations, PART 121--OPERATING REQUIREMENTS: DOMESTIC, FLAG, AND SUPPLEMENTAL OPERATIONS 121.401 Training Program: General and 121.418 Differences Training: Crew members and dispatchers, www.faa.gov

10 Marsh, "Is Currency Any Easier?" AOPA Magazine, AOPA, Feb. 1998.

${ }^{11}$ FAA, Aviation Instructor's Handbook, FAA-H-8083-9, ISBN 1560273828, ASA Inc, 2000

12 ibid, sec. 5-2

${ }^{13}$ Dept. of Health and Human Services, Code of Federal Regulations, TITLE 45: PUBLIC WELFARE, PART 46: PROTECTION OF HUMAN SUBJECTS 46.116 a) 1.June, 2005. 